\title{
BMJ Open Barriers to and facilitators of the implementation of pharmacist services in primary care clinics: a scoping review protocol
}

\author{
Haixin Li, ${ }^{1}$ Xujian Liang, ${ }^{2}$ Yang Wang (D) ${ }^{3}$ Yiting Lu, ${ }^{4}$ Zhiling Deng, ${ }^{5}$ Yuanqu Ye, ${ }^{6}$ \\ Yi Qian, ${ }^{7}$ Yi Guo, ${ }^{8}$ Zhijie Xu (D) ${ }^{8}$
}

To cite: Li H, Liang X, Wang Y, et al. Barriers to and facilitators of the implementation of pharmacist services in primary care clinics: a scoping review protocol. BMJ Open 2022;12:e057191. doi:10.1136/ bmjopen-2021-057191

- Prepublication history and additional supplemental material for this paper are available online. To view these files, please visit the journal online (http://dx.doi.org/10.1136/ bmjopen-2021-057191).

Received 08 September 2021 Accepted 22 December 2021

D) Check for updates

C Author(s) (or their employer(s)) 2022. Re-use permitted under CC BY-NC. No commercial re-use. See rights and permissions. Published by BMJ.

For numbered affiliations see end of article.

Correspondence to

Dr Zhijie Xu; zhijiexu@zju.edu.cn

\section{ABSTRACT}

Introduction Inappropriate medication use is a leading cause of avoidable harm in health systems and is particularly severe in primary care settings. Evidence has shown that the integration of pharmacists into primary care clinics has favourable satisfaction and effectiveness in health outcomes. However, barriers to and facilitators of pharmacist services in these settings have not been comprehensively reviewed. Therefore, this scoping review aims to map and examine the literature available on the barriers to and facilitators of the implementation of pharmacist services in primary care clinics to guide future implementation research.

Methods and analysis This scoping review will be undertaken following the six-stage framework developed by Arksey and 0'Malley and be guided by recommendations by Levac et al. Eight electronic databases (PubMed, Embase, Scopus, Web of Science, CINAHL, PsycINFO, CNKI and Wanfang) will be searched. Reference lists and related citations, and grey literature from websites will be searched manually. Available information that has been reported in Chinese or English up to 31 August 2021 will be included. Studies will be selected and screened by two reviewers independently. Findings from the included studies will be extracted by two independent reviewers and supervised by a third reviewer. A content analysis of the findings will be performed using MAXQDA 2020.

Ethics and dissemination Ethical approval will not be required for this scoping review, as all data and information will be obtained from publicly available literature. The findings of this scoping review will be shared with healthcare managers in primary care institutions and health authorities as well as disseminated via publication in a peer-reviewed journal.

\section{INTRODUCTION}

Inappropriate medication use poses a significant risk to individual health and is a leading cause of avoidable harm in health systems. The proportion of people hospitalised due to adverse drug events has been estimated at $6 \%$ in the USA, ${ }^{1}$ and approximately 197000 deaths annually have been caused by adverse
Strengths and limitations of this study

- This study will be the first scoping review aimed at synthesising barriers to and facilitators of the implementation of pharmacist services in primary care clinics.

- The study will employ a systematically designed search strategy to search eight databases and grey literature to ensure the comprehensiveness of the searched work.

- This scoping review will only consider studies published in English and Chinese and may overlook relevant studies in other languages.

- A critical appraisal of the study's quality and risk-ofbias assessment will not be undertaken, as this is a scoping review.

drug reactions (ADRs) in Europe. ${ }^{2}$ In addition, preventable ADRs cost $€ 2851-€ 9015$ per hospitalisation in the US and European countries. ${ }^{3}$ Inappropriate medication use in primary care settings has also been a matter of concern.

One way to reduce medication-related harm is the use of pharmacist services, which have been a key strategy for optimising medicine use and improving health outcomes. ${ }^{4-6}$ Previous studies have indicated that pharmacists have been integrated into primary care clinics to improve the quality of their services. ${ }^{7-11}$ The collaboration between pharmacists and health professionals in primary care clinics plays an important role in medicine management services. ${ }^{12}{ }^{13}$ Some studies have explored the satisfaction and effectiveness of incorporating pharmacist services into primary care clinics, reporting favourable results in the management of chronic diseases and quality use of medications. ${ }^{8}$ Prior studies have shown that the number of patient risk-factors for medication-related problems decreased significantly after the 
multifaceted intervention of pharmacist-led medication consultations and reviews in general practice clinics. ${ }^{14}$

Pharmacist integration in the primary care clinics can fill gaps in care and help ensure achievement of quality measure benchmarks. ${ }^{15}$ This clinical and financial impacts in turn facilitate the collaboration between pharmacists and other health professionals in primary care. ${ }^{15}$ However, primary care institutions in many areas are inadequate in terms of infrastructure, manpower and funding. ${ }^{16}$ The mechanism for the sustainable development of pharmacist services in primary care clinics remains immature, thus limiting its large-scale expansion. Previous qualitative studies have identified some barriers to and facilitators of the integration of pharmacists into general practice clinics. ${ }^{17} 18$ Their results demonstrated that ineffective inter-professional communication, lack of time and poor access to patient health records were barriers, ${ }^{17}$ whereas colocation and the interdisciplinary environment in primary care settings were identified as facilitators. ${ }^{18}$

To the best of our knowledge, barriers to and facilitators of pharmacist services to primary care patients in primary care clinics have not been comprehensively reviewed. Therefore, our scoping review aims to undertake a broad examination of published articles and grey literature regarding pharmacist services, focusing on barriers to and facilitators of services provided by pharmacists in primary care clinics. The key research question is as follows: 'What are the existing barriers to and facilitators of the implementation of pharmacist services in primary care clinics?'

\section{METHODS AND ANALYSIS}

This protocol was guided by the Preferred Reporting Items for Systematic Reviews and Meta-Analyses Extension for Scoping Reviews (PRISMA-ScR). ${ }^{19}$ The draft protocol was reviewed by all members of a multidisciplinary team and revised after discussion. The PRISMA-ScR checklist is presented in online supplemental file 1 . We plan to use the scoping review as the methodology of this study because it can examine and synthesise evidence from any research methodology, including nonresearch sources, in a given area. ${ }^{20}$ Thus, scoping reviews potentially provide us with a comprehensive and useful overview to clarify key concepts, inform future research and address knowledge gaps. We assembled an interdisciplinary team (HL, a licensed pharmacist; XL, YL and ZX, primary care physicians; ZD and YY, healthcare managers in community health centres; YW, a scientific editor of a primary care academic journal; and $\mathrm{YQ}$, a health policy researcher) to undertake this scoping review from the perspectives of pharmaceutical care, primary healthcare, health administration and scientific methodology.

This scoping review will be undertaken guided by the framework developed by Arksey and O'Malley, which includes the following six stages: (1) identifying the research question; (2) identifying relevant studies; (3) selecting studies; (4) charting the data; (5) collating, summarising and reporting results; and (6) consulting with stakeholders. ${ }^{21}$ We will also refer to recommendations by Levac et al that clarify and enhance each stage of the framework. ${ }^{22}$

\section{Stage 1: identifying the research question}

Scoping reviews require a broad research question and focus on summarising a breadth of evidence. ${ }^{22}$ The following overarching research question, which needs to be addressed by this scoping review, was discussed and formulated by reviewers from the multidisciplinary team: 'What are the existing barriers to and facilitators of the implementation of pharmacist services in primary care clinics?' The rationale behind this research question is that despite pharmacist services becoming increasingly important in primary care settings, their implementation faces challenges in many aspects and they have not been widely developed. The target population of interest includes licensed pharmacists who work wholly or partly in primary care clinics. Pharmacist services that do not aim to optimise medication use, such as the dispensing of drugs, will not be included. The context is limited to clinics in primary care settings; thus, nursing homes and long-term facilities will not be considered because they usually provide inpatient care. Barriers and facilitators will be considered participant-reported or researcherinterpreted determinants that may impede or improve the implementation and development of pharmacist services in primary care clinics.

\section{Stage 2: identifying relevant studies}

We will conduct a comprehensive search of articles and grey literature published up to 31 August 2021 in English and Chinese. First, we will identify studies using relevant text words and medical headings in eight electronic databases, namely, PubMed, Embase, Scopus, Web of Science, CINAHL, PsycINFO, CNKI and Wanfang. The initial search strategy will be piloted to verify its breadth, comprehensiveness and feasibility. The key concepts and PubMed search terms are listed in table 1. Second, we will conduct a grey literature search from websites of relevant organisations for available information to achieve the level of comprehensiveness required for a scoping review. ${ }^{23}$ The organisations include the WHO, pharmaceutical associations worldwide, Google Scholar, New York Academy of Medicine Grey Literature Report, and other community health institutions. Third, we will review the reference lists of included studies to identify relevant studies that would not have been identified in the initial search. This search strategy will be applied to every database. The full search strategies for all databases and websites are presented in online supplemental file 2. An additional search will be conducted using updated search terms if any other search terms are discovered during the search. Search results will be sorted in Microsoft Excel, and duplicates will be removed before screening and data extraction. 
Table 1 List of search strategy in terms of text words and medical headings

\begin{tabular}{|c|c|c|c|}
\hline & Population & Concept & Context \\
\hline \multirow[t]{9}{*}{ Text words } & Pharmacist & Pharmacist services & Primary care clinics \\
\hline & Clinical pharmacist & Pharmaceutical care & Family practice \\
\hline & Pharmacy staff & Pharmacy clinic for outpatients & Community Health Centers \\
\hline & Pharmaceutics & Pharmacy clinic & Community Hospitals \\
\hline & Apothecary & Pharmacology services & Pharmacist-managed clinics \\
\hline & Hospital pharmacist & Outpatient pharmaceutical service & Primary care settings \\
\hline & Assistant pharmacist & Outpatient pharmacy service & Ambulatory care settings \\
\hline & Pharmacy technician & Barriers & Clinics in general practice \\
\hline & Primary healthcare professionals & Facilitators & Pharmacist-led medication review clinics \\
\hline \multirow{9}{*}{ PubMed } & Pharmacy Technicians (MeSH) & Pharmaceutical Services (MeSH) & Community Health Centers (MeSH) \\
\hline & Pharmac $^{\star}$ technician & Pharmac $^{\star}$ servic $^{*}$ & Community health services (MeSH) \\
\hline & Pharmacist* & Pharmac* care & Primary care clinic* \\
\hline & Clinic $^{*}$ pharmacist $^{\star}$ & Pharmacist-managed clinic* & Pharmacist-managed clinic* \\
\hline & Primary care pharmacist* & Pharmac ${ }^{\star}$ clinic for outpatient ${ }^{\star}$ & Community health servic* \\
\hline & Pharmac* staff & Pharmac $^{*}$ clinic* $^{*}$ & Commun* Health Center ${ }^{*}$ \\
\hline & Pharmaceutist $^{\star}$ & outpatient pharmac ${ }^{\star}$ servic $^{\star}$ & Commun $^{*}$ Hospital $^{*}$ \\
\hline & Druggist* $^{*}$ & Facilitator $^{\star}$ & General practice* $^{*}$ \\
\hline & Apothecary* & Barrier* & Community care setting ${ }^{*}$ \\
\hline
\end{tabular}

\section{Stage 3: study selection}

In this stage, a transparent and replicable process will be used by our team, and it will involve searching the literature, refining the search strategy and reviewing articles for study inclusion. Before the screening process commences, all reviewers will undergo training to improve the inter-rater reliability of this scoping review. The challenges and uncertainties of the study selection will be discussed during the entire process of study selection, and the search strategy will be refined if necessary. Articles identified from the electronic databases and other sources will be independently reviewed to screen the titles and abstracts by two reviewers (HL and XL) using a structured screening tool, which will be pilot tested on a group of randomly selected titles and abstracts to ensure high consistency and reliability. Subsequently, the two reviewers will screen the full text of identified articles thoroughly and exclude irrelevant articles based on the eligibility criteria. They will discuss and reach a consensus regarding eligibility, and a third reviewer (ZX) will be consulted to resolve discrepancies. The PRISMA flow diagram (figure 1) will be used to report the search results and record the reasons for the excluded articles. We will update the flow diagram on completion of the scoping review.

\section{Eligibility criteria}

An article will be included if it:

1. Aimed to examine barriers to or facilitators of pharmacist services.

2. Was conducted in primary care clinics.

3. Was published in English or Chinese.

4. Was published before 31 August 2021.

5. Was a full report of an original research.

An article will be excluded if it used no research method to examine barriers and facilitators (eg, letters to the editor, editorials, reviews, comments). It should be noted that reviewers may search the reference lists of excluded articles to ensure the comprehensiveness of our scoping review. Regarding studies that were conducted in both 


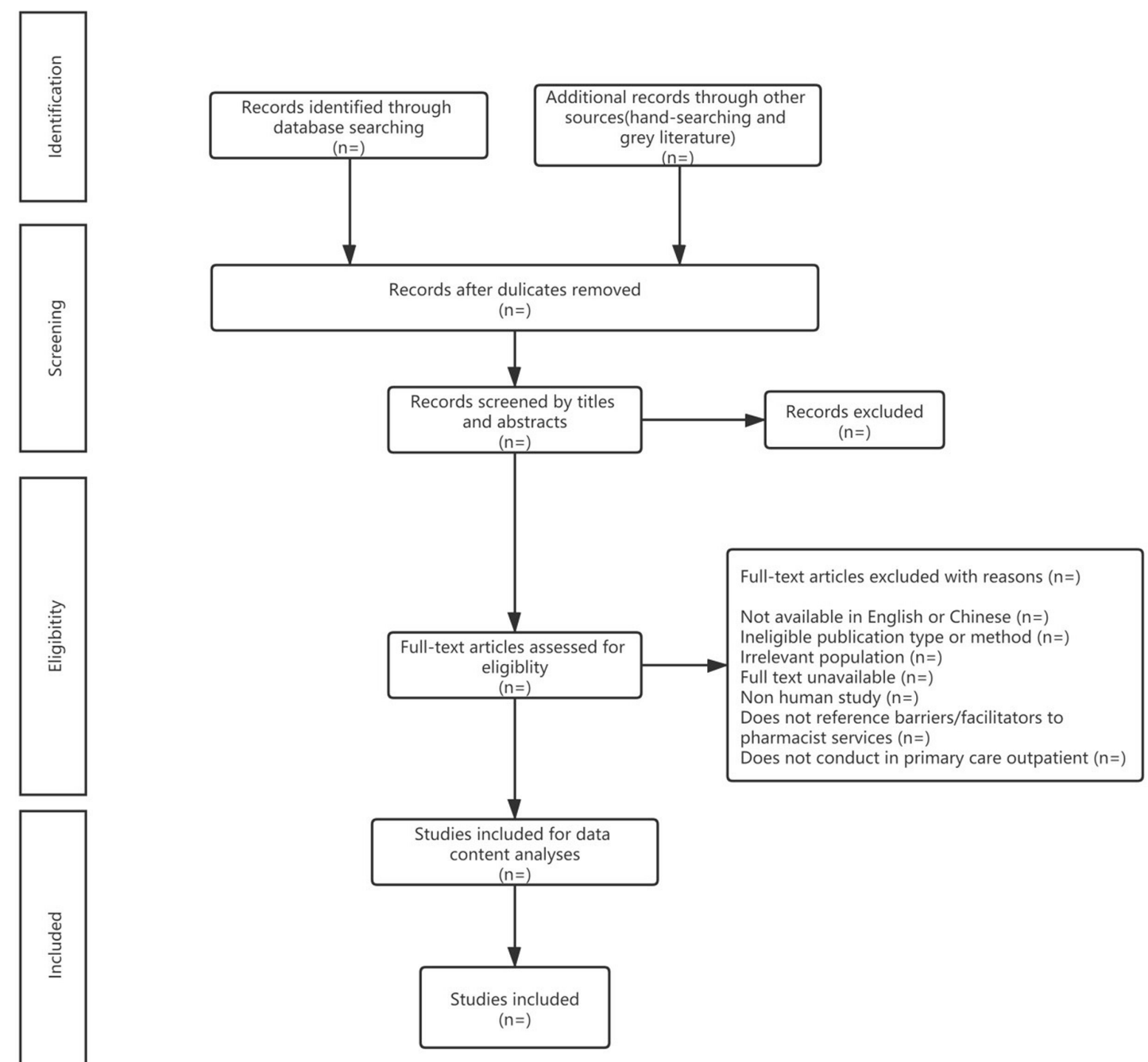

Figure 1 PRISMA Extension for Scoping reviews, 2018 flow diagram. PRISMA, Preferred Reporting Items for Systematic Reviews and Meta-Analyses.

primary care clinics and other settings concurrently, only findings specific to primary care clinics will be extracted and analysed.

\section{Stage 4: charting the data}

A standardised data-charting form will initially be developed and piloted by the reviewer team through an iterative process. Before charting the data formally, we will perform a trial data-charting exercise. Two reviewers (HL and XL) will independently extract the data from the same 10 studies to ensure that the approach to data extraction is consistent with the research question. We will chart the data formally until $80 \%$ inter-rater agreement is achieved across all categories. Data will be extracted from the following categories: first author's name, journal name, year of publication, geographic location, study setting, study period, study design, research tools, sample size, participant characteristics and main findings regarding barriers to and facilitators of pharmacist services in primary care clinics. In cases where data in the selected articles are unclear or missing, we will contact the study authors via email. Discrepancies between the two reviewers in this process will be resolved through discussion, and a third reviewer (ZX) will be consulted if disagreement persists.

\section{Stage 5: collating, summarising and reporting the results}

All selected studies will be entered verbatim into MAXQDA 2020. This stage will occur in three phases. First, we will analyse the data using a descriptive numerical summary to describe the characteristics of the included studies and apply a content analysis approach to identify barriers to and facilitators of pharmacist services in primary care clinics. We will employ MAXQDA 2020 to conduct the content analysis. ${ }^{24}$ Two reviewers (YL and HL) will undergo training on coding the extracted data using a broad-based coding scheme to achieve $80 \%$ coding agreement. Second, we will report the analysed results using themes and produce the outcomes with reference to our study purpose. Third, we will perform an overall interpretation of the relationships among the synthesised themes and subthemes and of the meaning of our findings as well as identifying the knowledge gaps. Implications for future research, clinical practice and policy-making will also 
be discussed. Consistent with the framework proposed by Arksey and O'Malley, an assessment of the quality of individual studies and a risk-of-bias assessment will not be conducted.

\section{Stage 6: consultation with stakeholders}

Stakeholder consultation, though considered an optional exercise in Arksey and O'Malley's framework, ${ }^{21}$ could provide additional valuable insights into the literature review regarding practical problems faced by pharmacist services and opportunities for knowledge transfer in the field. After completing the collating, summarising and reporting of results (stage 5), we will formulate a detailed design of the consultation process and engage relevant stakeholders, including pharmacists and other primary care practitioners in community health centres, with or without administrative tasks. We will also approach patients who would have received pharmacist services in primary care clinics. We will share preliminary findings from stage 5 with stakeholders and conduct in-depth interviews and conventional content analysis to elicit and analyse stakeholders' perspectives regarding barriers and facilitators not yet published. ${ }^{24}$ For instance, we will hold the group meetings with stakeholders to share and discuss the results of our scoping review. Besides, we will sort out the original data and categorise the views of different groups from the articles included. These synthesised results will be shared with relevant stakeholders (eg, primary care physicians, primary care clinical pharmacists, healthcare managers), respectively.

\section{Patient and public involvement}

We did not involve patients in the preparation of this protocol, and they will not be involved in the final scoping review. However, we selected two primary care clinical pharmacists and one director of pharmacy from two community health centres in Hangzhou and Shenzhen who agreed to determine the research agenda, developing the protocol and interpreting and reporting the results. We will also share the synthesised results with primary care clinical pharmacists through international cooperation, who will help us assess whether the context of the articles reviewed are interpreted and considered accurately. The primary care clinical pharmacists in multiple countries will examine the results to ensure the rigour and reproducibility of the results. Additionally, three authors (YL, YY and ZD) will work closely with pharmacists in community health centres and provide guidance on designing the scoping review.

\section{ETHICS AND DISSEMINATION}

Ethical approval is not required for this scoping review, as all data and information will be obtained from publicly available literature and will not involve animals or human participants. The findings of this scoping review will be shared with healthcare managers in primary care institutions and health authorities as well as disseminated via publication in a peer-reviewed journal. Although the presence of pharmacists in primary care clinics providing pharmaceutical care is not widespread in many countries, including China, efforts have been made to explore a feasible approach. This scoping review is a fundamental work in a multistage research project that aims to develop pharmacist services in primary care settings. We anticipate that our findings will contribute to the literature a diversity of perspectives regarding the multiple levels of barriers to and facilitators of the implementation of pharmacist services in primary care clinics. These may help guide the direction of future research, inform policy-makers and organisation leaders in addressing barriers to the implementation of pharmacist services, and aid clinical pharmacists working in primary care clinics.

\section{Author affiliations}

${ }^{1}$ School of Pharmacy, Lanzhou University, Lanzhou, China

${ }^{2}$ Department of General Practice, Sir Run Run Shaw Hospital, Zhejiang University School of Medicine, Hangzhou, China

${ }^{3}$ Chinese General Practice Press, Beijing, China

${ }^{4}$ Zhongdai Community Healthcare Center, Huzhou, China

${ }^{5}$ Cuihai Community Healthcare Center, The Eighth Affiliated Hospital, Sun Yat-sen University, Shenzhen, China

${ }^{6}$ Baili Community Healthcare Center, The People's Hospital of Longhua, Shenzhen, China

${ }^{7}$ School of Public Health, Hangzhou Normal University, Hangzhou, China

${ }^{8}$ Department of General Practice, The Second Affiliated Hospital, Zhejiang University School of Medicine, Hangzhou, China

Contributors ZX had the original idea of this study. XL contributed to the development of this idea. HL developed the first version of the protocol and search strategies with support from the ZX. YW and YQ reviewed the manuscript for intellectual content and contributed to refining the search strategy and eligibility criteria. ZD, YY, YL and YG read and critiqued the draft, and ZX performed the final review of the manuscript and submitted it. All the authors approved the final version of the manuscript.

Funding This work was supported by the Scientific Research Fund of Zhejiang Provincial Education Department (grant number: Y201941691) and the National Natural Science Foundation of China (grant number: 72004050).

Competing interests None declared.

Patient consent for publication Not applicable.

Provenance and peer review Not commissioned; externally peer reviewed.

Supplemental material This content has been supplied by the author(s). It has not been vetted by BMJ Publishing Group Limited (BMJ) and may not have been peer-reviewed. Any opinions or recommendations discussed are solely those of the author(s) and are not endorsed by BMJ. BMJ disclaims all liability and responsibility arising from any reliance placed on the content. Where the content includes any translated material, BMJ does not warrant the accuracy and reliability of the translations (including but not limited to local regulations, clinical guidelines, terminology, drug names and drug dosages), and is not responsible for any error and/or omissions arising from translation and adaptation or otherwise.

Open access This is an open access article distributed in accordance with the Creative Commons Attribution Non Commercial (CC BY-NC 4.0) license, which permits others to distribute, remix, adapt, build upon this work non-commercially, and license their derivative works on different terms, provided the original work is properly cited, appropriate credit is given, any changes made indicated, and the use is non-commercial. See: http://creativecommons.org/licenses/by-nc/4.0/.

\section{ORCID iDs}

Yang Wang http://orcid.org/0000-0001-6523-7517

Zhijie Xu http://orcid.org/0000-0003-4824-6176 


\section{REFERENCES}

1 Poudel DR, Acharya P, Ghimire S, et al. Burden of hospitalizations related to adverse drug events in the USA: a retrospective analysis from large inpatient database. Pharmacoepidemiol Drug Saf 2017;26:635-41.

2 Bouvy JC, De Bruin ML, Koopmanschap MA. Epidemiology of adverse drug reactions in Europe: a review of recent observational studies. Drug Saf 2015;38:437-53.

3 Formica D, Sultana J, Cutroneo PM, et al. The economic burden of preventable adverse drug reactions: a systematic review of observational studies. Expert Opin Drug Saf 2018;17:681-95.

$4 \mathrm{Xu} \mathrm{Z}$, Liang X, Zhu Y, et al. Factors associated with potentially inappropriate prescriptions and barriers to medicines optimisation among older adults in primary care settings: a systematic review. Fam Med Com Health 2021;9:e001325.

5 De Barra M, Scott CL, Scott NW. Pharmacist services for non-hospitalised patients. Cochrane Database Syst Rev 2018;9:CD013102.

6 Nkansah N, Mostovetsky O, Yu C. Effect of outpatient pharmacists' non-dispensing roles on patient outcomes and prescribing patterns. Cochrane Database Syst Rev 2010;7:CD000336.

7 Lui E, Ha R, Truong C. Applying the pharmaceutical care model to assess pharmacist services in a primary care setting. Can Pharm $J$ 2017;150:90-3.

8 Tan ECK, Stewart K, Elliott RA, et al. Pharmacist services provided in general practice clinics: a systematic review and meta-analysis. Res Social Adm Pharm 2014;10:608-22.

9 Petty DR, Knapp P, Raynor DK. Patients' views of a pharmacistrun medication review clinic in general practice. Br J Gen Pract 2003;53:607-13.

10 MacRae F, Lowrie R, MacLaren A. Pharmacist-led medication review clinics in general practice: the views of greater Glasgow. Int J Pharm Pract 2014;22:28-37.

11 Norman JL, Kroehl ME, Lam HM, et al. Implementation of a pharmacist-managed clinic for patients with chronic nonmalignant pain. Am J Health Syst Pharm 2017;74:1229-35.
12 Rahayu SA, Widianto S, Defi IR, et al. Role of pharmacists in the interprofessional care team for patients with chronic diseases. $J$ Multidiscip Healthc 2021;14:1701-10.

13 Foot H, Scott I, Sturman N. Impact of pharmacist and physician collaborations in primary care on reducing readmission to hospital: a systematic review and meta-analysis. Res Social Adm Pharm 2021;Jul 16:S1551-7411:00273-4.

14 Tan ECK, Stewart K, Elliott RA, et al. Pharmacist consultations in general practice clinics: the pharmacists in practice study (PIPs). Res Social Adm Pharm 2014;10:623-32.

15 Yon K, Sinclair J, Bentley OS, et al. Impact of quality measures performed through pharmacist collaboration with a primary care clinic. J Am Pharm Assoc 2020;60:S97-102.

16 Smith MA. Implementing primary care pharmacist services: go upstream in the world of value-based payment models. Res Social Adm Pharm 2017;13:892-5.

17 Tan ECK, Stewart K, Elliott RA. Stakeholder experiences with general practice pharmacist services: a qualitative study. BMJ Open 2013;3:e003214.

18 Tan ECK, Stewart K, Elliott RA, et al. Integration of pharmacists into general practice clinics in Australia: the views of general practitioners and pharmacists. Int J Pharm Pract 2014;22:28-37.

19 Tricco AC, Lillie E, Zarin W, et al. PRISMA extension for scoping reviews (PRISMA-ScR): checklist and explanation. Ann Intern Med 2018;169:467-73.

20 Peters MDJ, Marnie C, Tricco AC, et al. Updated methodological guidance for the conduct of scoping reviews. JBI Evid Implement 2021;19:3-10.

21 Arksey H, O'Malley L. Scoping studies: towards a methodological framework. Int J Soc Res Methodol 2005;8:19-32.

22 Levac D, Colquhoun H, O'Brien KK. Scoping studies: advancing the methodology. Implement Sci 2010;5:69.

23 Godin K, Stapleton J, Kirkpatrick SI, et al. Applying systematic review search methods to the grey literature: a case study examining guidelines for school-based breakfast programs in Canada. Syst Rev 2015;4:138.

24 Hsieh H-F, Shannon SE. Three approaches to qualitative content analysis. Qual Health Res 2005;15:1277-88. 